IPMU 08-0021

KEK-TH-1243

\title{
Upperbound on Squark Masses in Gauge-Mediation Model with Light Gravitino
}

\author{
Junji Hisano $^{(a, b)}$, Minoru Nagai ${ }^{(c)}$, Shohei Sugiyama ${ }^{(a)}$, and T. T. Yanagida ${ }^{(d, b)}$ \\ (a) Institute for Cosmic Ray Research (ICRR), \\ University of Tokyo, Kashiwa, Chiba 277-8582, Japan \\ (b) Institute for the Physics and Mathematics of the Universe (IPMU), \\ University of Tokyo, Kashiwa, Chiba 277-8568, Japan \\ (c) Theory Group, KEK, Oho 1-1, Tsukuba, Ibaraki 305-0801, Japan \\ (d) Department of Physics, University of Tokyo, \\ Tokyo 113-0033, Japan
}

\begin{abstract}
We examine stabilities of our supersymmetry-breaking false vacuum in a lowenergy direct gauge mediation model of SUSY breaking. The stability required in the high-temperature early universe leads to upperbounds on masses of squarks and gluino as $m_{\tilde{q}} \lesssim 1 \mathrm{TeV}$ and $m_{\tilde{g}} \lesssim 1 \mathrm{TeV}$ for the light gravitino of mass $m_{3 / 2} \lesssim 16 \mathrm{eV}$.
\end{abstract}




\section{Introduction}

The light gravitino of mass $\lesssim 16 \mathrm{eV}$ is very attractive, since it causes no astrophysical and cosmological [1, 2, 3] gravitino problem. The gauge mediation [4] is only a known mechanism to have such a light gravitino with a consistent spectrum for supersymmetry (SUSY) particles. The light gravitino requires relatively light messenger quarks and leptons at $O(100) \mathrm{TeV}$ for generating SUSY particles of mass $O(1) \mathrm{TeV}$. This is an attractive point for future high-energy experiments. In addition, the latest experimental data on the anomalous magnetic moment of the muon and on the branching ratio of $\bar{B} \rightarrow X_{s} \gamma$ favor a low messenger scale $(\sim 100 \mathrm{TeV})$ [5]. However, on the other hand, the presence of the light messengers may cause a serious problem in cosmology, since it often generates a SUSY-invariant true vacuum near the SUSY-breaking false one.

If there is indeed a SUSY-invariant true vacuum near our SUSY-breaking false vacuum, the vacuum transition to the true one should be sufficiently suppressed at least in the present universe. In a recent article [6], constraints from the quantum vacuum transition have been derived in a generic minimal gauge-mediation model. However, one should also consider the thermal vacuum transition if the universe underwent through a high temperature regime. The purpose of this paper is to discuss the vacuum transition at high temperatures in the early universe by assuming an explicit model for the gauge mediation. For this purpose we take the IYIT SUSY-breaking model [7], since it easily accommodates a gauge-mediation model with the light gravitino.

In this paper we evaluate both the quantum and thermal transition rates of the SUSYbreaking to SUSY-invariant true vacua in the model. We derive upperbounds on squark

and gluino masses $\left(m_{\tilde{q}} \lesssim 1 \mathrm{TeV}\right.$ and $\left.m_{\tilde{g}} \lesssim 1 \mathrm{TeV}\right)$ for the stability of our SUSY-breaking vacuum in the early universe. The bounds are given in the minimal messenger model. We also briefly discuss the upperbounds on masses for the SUSY particles in possible extensions of the minimal model. 


\section{Gauge-mediation model with light gravitino}

Our model is based on the IYIT model [7] for SUSY breaking. We introduce a hidden $S U(2)$ gauge group with four chiral superfields $Q_{i}(i=1 \ldots 4)$ transforming as fundamental representations of $S U(2)$. We add singlets $S^{i j}\left(=-S^{j i}\right)$ in 6 -dimensional representation of the flavor $S U(4)$ symmetry of our model. This particle content is minimal in the IYIT SUSY-breaking models. The superpotential is given by $W=-\frac{1}{2} \lambda S^{i j} Q_{i} Q_{j}$. At the low energy this theory is in the confining phase and the moduli space is modified by the strong dynamics as, $\operatorname{Pf}\left(Q_{i} Q_{j}\right)=\Lambda^{4}$, where $\Lambda$ denotes the holomorphic dynamical scale.

The low-energy effective superpotential is dictated with singlets $S^{i j}$ and mesons $M_{i j} \simeq$ $Q_{i} Q_{j}$. It is convenient to exploit the local equivalence of $S U(4)$ and $S O(6)$ for the flavor symmetry, and to regard both singlets and mesons to be in the vector representations of $S O(6), S_{a}$ and $M_{a}(a=0 \cdots 5)$. In those representations, the quantum modified moduli space and superpotential are given as

$$
\begin{gathered}
\operatorname{Pf}(M)=M_{a} M_{a}=\Lambda^{2}, \\
W_{\text {eff }}=-\lambda \Lambda S_{a} M_{a},
\end{gathered}
$$

respectively. Here we have rescaled by factor of $\Lambda$ so that $M_{a}$ has mass dimension +1 . By solving the constraint of the quantum moduli space, we get

$$
W_{\mathrm{eff}}=-\lambda \Lambda S \sqrt{\Lambda^{2}-M_{a}^{\prime} M_{a}^{\prime}}-\lambda \Lambda S_{a}^{\prime} M_{a}^{\prime},
$$

where $S=S_{0}$ and $M_{a}^{\prime}=M_{a}$ and $S_{a}^{\prime}=S_{a}$ for $a=1 \cdots 5$. SUSY is dynamically broken with

$$
F_{S}\left(\equiv \mu^{2}\right)=\lambda \Lambda^{2}
$$

The most important ingredient for our purpose is the form of Kahler potential. The integration of the light mesons $M_{a}^{\prime}$ and singlets $S_{a}^{\prime}$ gives rise to the effective Kahler potential,

$$
K=S^{\dagger} S-\frac{\eta}{4 \Lambda^{2}}\left(S^{\dagger} S\right)^{2}+\cdots,
$$

around $S \lesssim 4 \pi \Lambda$. The parameter $\eta$ is [8]

$$
\eta \simeq \frac{5 \lambda^{2}}{16 \pi^{2}}(2 \log 2-1)
$$


at the one-loop level. The second term in Eq. (5) gives a mass term to the scalar component of $S$ as $m_{S}^{2}=\eta\left|F_{s}\right|^{2} / \Lambda^{2}=\eta \lambda \mu^{2}$, and it makes the SUSY-breaking vacuum classically stable around $S \simeq 0$ since $\eta>0$. Other hadron states also contribute to the Kahler potential, which is, however, uncalculable due to the strong dynamics of the hidden $S U(2)$. Fortunately, it is found, using the naive dimensional analysis, that the contribution from the light mesons and singlets to $m_{S}^{2}$ in Eq. (15) dominates over those from the uncalculable hadron integrations [8].

We introduce a minimal messenger sector. It contains only a pair of messenger quarks and leptons, $\Phi_{d}+\bar{\Phi}_{d}$ and $\Phi_{l}+\bar{\Phi}_{l}$, where they belong to $5+5^{\star}$ in the $S U(5)_{\mathrm{GUT}}$, together. We do not introduce more messengers, since it may cause too large $\mathrm{CP}$ violation. In this sense, an alternative choice of the messengers is a pair of messengers belonging to $10+10^{\star}$. We will shortly discuss effects of this choice later.

The superpotential for the messengers $\Phi_{i}$ and $\bar{\Phi}_{i}(i=d, l)$ is

$$
W=\left(\kappa_{d} S-M_{d}\right) \Phi_{d} \bar{\Phi}_{d}+\left(\kappa_{l} S-M_{l}\right) \Phi_{l} \bar{\Phi}_{l}
$$

In this paper we take $M_{d}=M_{l}(\equiv M)$ and $\kappa_{d}=\kappa_{l}(\equiv \kappa)$ for simplicity. One-loop diagrams generate gaugino masses in the SUSY standard model (SSM), $M_{\tilde{g}_{i}}(i=1-3)$, as

$$
M_{\tilde{g}_{i}}=\frac{\alpha_{i}}{4 \pi} \frac{\kappa \mu^{2}}{M} g(x),
$$

and the two-loop diagrams give sfermion masses $m_{\tilde{f}}$ as

$$
m_{\tilde{f}}^{2}=2\left[C_{3}^{\tilde{f}}\left(\frac{\alpha_{3}}{4 \pi}\right)^{2}+C_{2}^{\tilde{f}}\left(\frac{\alpha_{2}}{4 \pi}\right)^{2}+\frac{3}{5} Y_{\tilde{f}}^{2}\left(\frac{\alpha_{1}}{4 \pi}\right)^{2}\right]\left(\frac{\kappa \mu^{2}}{M}\right)^{2} f(x)
$$

where $C_{3}^{\tilde{f}}, C_{2}^{\tilde{f}}$ and $Y_{\tilde{f}}$ are $S U(3)_{C}$ and $S U(2)_{L}$ quadratic Casimirs and hypercharge of sfermion $\tilde{f}$, respectively. The mass functions $f(x)$ and $g(x)$ depend on $x \equiv \kappa \mu^{2} / M^{2}$, and their explicit forms are given in Ref. [9].

Now we discuss classical stability of the SUSY-breaking vacuum in our model. Introduction of the messengers generates the SUSY-invariant true vacuum at

$$
S \simeq \frac{M}{\kappa}, \quad \Phi_{d} \bar{\Phi}_{d}+\Phi_{l} \bar{\Phi}_{l}=\frac{\mu^{2}}{\kappa} .
$$


The SUSY-breaking false vacuum is, however, classically stable under following two conditions. First, masses for the bosonic components of the messengers are given by $M^{2} \pm \kappa \mu^{2}$ around $S \simeq 0$. Then, the SUSY-breaking vacuum is classically stable when

$$
M^{2} \gtrsim \kappa \mu^{2}
$$

Second, the mass terms of the messengers in Eq. (7) break the $U(1)_{R}$ symmetry, and it generates corrections to the scalar potential for $S$, which may make the SUSY-breaking vacuum unstable [10]. One-loop diagrams with the messengers give the corrections to the scalar potential for $S$ as

$$
\delta V \simeq-\frac{5 \kappa^{2} \mu^{4}}{16 \pi^{2}}\left[\frac{\kappa}{M}\left(S+S^{\dagger}\right)+\frac{\kappa^{2}}{2 M^{2}}\left(S^{2}+S^{\dagger 2}\right)+\cdots\right] .
$$

The SUSY-breaking minimum is sifted toward the SUSY-invariant vacuum $\left(\sim(\kappa / \lambda)^{3} \times\right.$ $\left.\left(\mu^{2} / M\right)\right)$, and the false vacuum becomes destabilized unless

$$
\lambda^{3} \gtrsim \kappa^{4} \frac{\mu^{2}}{M^{2}}
$$

We see that the classical stability conditions of the SUSY-breaking false vacuum give a lowerbound on the gravitino mass. The gravitino mass is given by

$$
m_{3 / 2} \simeq \frac{F_{S}}{\sqrt{3} M_{\mathrm{pl}}} \simeq 10 \mathrm{eV}\left(\frac{m_{\tilde{q}}}{2 \mathrm{TeV}}\right)^{2}\left(\frac{M}{\kappa \mu}\right)^{2},
$$

where $M_{\mathrm{pl}}=2.4 \times 10^{18} \mathrm{GeV}$ is the reduced Planck mass. Here the mass scale is normalized by the squark mass $m_{\tilde{q}}$, which is approximately given by Eq. (9) with $f(x)=1$. This is because the mass function $\sqrt{f(x)}$ is less sensitive to $x$ than $g(x)(g(x)$ changes gradually from 1 to 1.4). It is found from Eqs. (11, 13) that the last factor in Eq. (14) is larger than $\sim \kappa^{-1}$ and also $\sim \kappa^{2} / \lambda^{3}$. Larger $\lambda$ is favored for a smaller gravitino mass. On the other hand, the reliability of our perturbative calculation requires $\lambda \lesssim 2$. Notice that if one imposes that the coupling $\lambda$ is small enough for perturbative calculation to be valid at the GUT scale and no extra $S U(2)$ multiplets are introduced, $\lambda$ should be smaller than $\sim 1$ at the SUSY-breaking scale. Thus, the light gravitino mass $m_{3 / 2}<16 \mathrm{eV}$ is already marginal even from a viewpoint of the classical stability conditions in our model.

In the following, we evaluate the quantum and thermal transition rates from the SUSYbreaking to SUSY-invariant true vacua, and show that there are severer constraints on the model parameters. 


\section{Transition to the true vacuum at zero temperature}

Let us estimate the quantum transition rate of the SUSY-breaking to SUSY-invariant vacua in our model by using the semiclassical approximation [11. Our model has several complex scalar fields. We first find a bounce solution by a numerical method in Ref. [12] and then estimate the four-dimensional Euclidean action $S_{4}$. For the technical details of our analysis, see Ref. [6].

We derive a bounce solution whose path is from the SUSY-breaking false vacuum $(S \sim$ 0 and $\left.\Phi_{d} \bar{\Phi}_{d}=\Phi_{l} \bar{\Phi}_{l}=0\right)$ to the SUSY-invariant true vacuum $\left(S=M / \kappa, \Phi_{d} \bar{\Phi}_{d}=0\right.$ and $\left.\Phi_{l} \bar{\Phi}_{l}=\mu^{2} / \kappa\right)$. We take $S$ and $\Phi_{l}=\bar{\Phi}_{l}$ real for simplicity, and other fields are vanishing. The minimalization of the $D$-term potential for messengers leads to this configuration $\left|\Phi_{l}\right|=\left|\bar{\Phi}_{l}\right|$, and the total potential for the direction along $\Phi_{l}=\bar{\Phi}_{l}$ becomes unstable when $S$ is increased. Defining $\phi_{1} \equiv \Phi_{l}=\bar{\Phi}_{l}$ and $\phi_{2} \equiv S$, we have the following $O(4)$ symmetric Euclidean action,

$$
S_{4}\left[\phi_{i}(r)\right]=2 \pi^{2} \int_{0}^{\infty} d r r^{3}\left[\sum_{i=1}^{2} \frac{k_{i}}{2}\left(\frac{d \phi_{i}}{d r}\right)^{2}+\left(V_{T=0}\left(\phi_{i}\right)-V_{T=0}\left(\phi_{i}^{f}\right)\right)\right],
$$

where $k_{1}=4$ and $k_{2}=2$, and $V_{T=0}\left(\phi_{i}\right)$ and $V_{T=0}\left(\phi_{i}^{f}\right)$ are the one-loop corrected effective scalar potential and the false vacuum energy at zero temperature, respectively. As explained in the previous section, the loop corrections of mesons $M_{a}^{\prime}$ and singlets $S_{a}^{\prime}$ stabilize the SUSY breaking vacuum at $S=0$, while those of the messengers destabilize it. Thus, the one-loop corrections to the scalar potential is very important and it should be included in our analysis.

The SUSY-invariant vacuum has a flat direction when $M_{d} / \kappa_{d}=M_{l} / \kappa_{l}$ in Eq. (77). This may enhance the prefactor $A$ in the transition rate per unit volume, $\Gamma / V=A \exp \left(-S_{4}\right)$. This situation is very similar to the tunnelings in systems with spontaneous symmetry breaking discussed in [13]. However, the possible enhancement would not be significant in the evaluation of the lowerbound on $S_{4}$. Here, we approximate the prefactor as $A \sim \mu^{4}$,

and $S_{4} \gtrsim 400$ is required to make the lifetime of the false vacuum much longer than the age of the universe.

In Fig 1 (left), we show contour plots for $S_{4}=400$ on a plane of $\kappa$ and $M / \kappa \mu$ for $\lambda=2,1,0.5$. The lines follow almost the boundary of the classical stability conditions of 

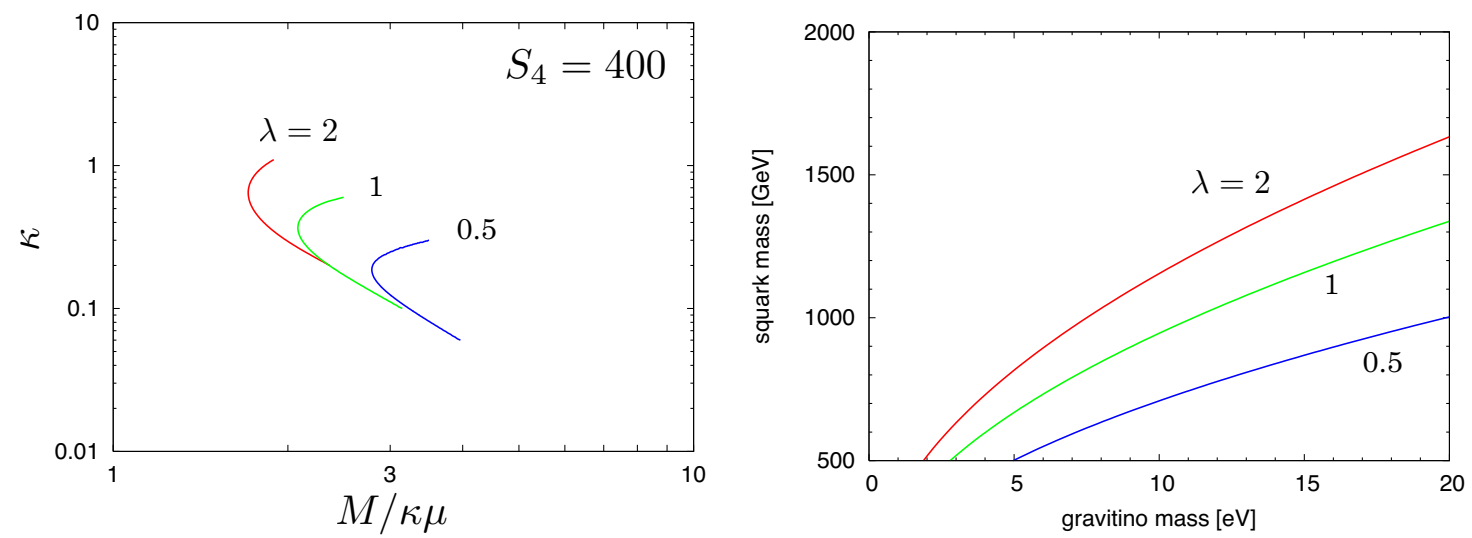

Figure 1: (Left) Contour plots for $S_{4}=400$ on a plane of $M / \kappa \mu\left(\mu^{2} \equiv \lambda \Lambda^{2}\right)$ and $\kappa$ for $\lambda=0.5,1,2$. On the right-handed sides of the lines $S_{4}$ is larger than 400. (Right) Squark mass upperbounds from quantum stability of the SUSY-breaking vacuum as functions of gravitino mass.

the SUSY-breaking vacuum in Eqs. (11, 13); $(M / \kappa \mu)^{-2} \lesssim \kappa \lesssim \lambda^{3 / 2}(M / \kappa \mu)$. Notice that larger $\lambda$ increases the mass squared $m_{S}^{2}$ and hence it makes smaller $M / \kappa \mu$ to be allowed.

In the parameter regions away from the lines toward the right, the parameter dependence of $S_{4}$ is understood from an approximate estimate based on Ref. [14]. In the approximation the vacuum transition rate is calculated for a triangle-shaped potential of one real scalar field. In our model, the true vacuum becomes more far from the false one for larger $M / \kappa \mu$, while the potential barrier is not high compared with the energy gap between true and false vacua. In this case, $S_{4}$ is approximately given as

$$
S_{4} \simeq 8 \pi^{2}\left(\frac{M}{\kappa \mu}\right)^{4} .
$$

Then, when $M / \kappa \mu$ is larger, the SUSY-breaking vacuum is more stable against the quantum vacuum transition.

In Fig 1 (right), the upperbounds on the squark masses are shown for several values of $\lambda$ as functions of the gravitino mass. It is found from Eq. (14) that smaller $M / \kappa \mu$ allows larger squark masses with $m_{3 / 2}$ fixed, while $M / \kappa \mu$ is bounded from below due to the quantum stability of the SUSY-breaking false vacuum. Thus, it is found that the squark masses have to be smaller than $1.5(1.2,0.9) \mathrm{TeV}$ for $\lambda=2(1,0.5)$ for $m_{3 / 2}<16 \mathrm{eV}$. 


\section{Thermal transition in the early universe}

Now, we discuss about thermal transitions of the SUSY-breaking to SUSY-invariant vacua in the early universe. At very high temperatures, the SUSY-breaking vacuum is automatically selected due to the thermal correction to the scalar potential in our model as shown below. However, it depends on various parameters in the model whether the false vacuum survives or not until temperature falls much below $\sim \mu$.

Let us explain the thermal history of the universe in our model. When the temperature of the universe $T$ falls below $\sim 4 \pi \Lambda$, the hidden $S U(2)$ gauge interaction in the SUSYbreaking sector becomes strong, and the $S U(2)$ quarks are confined so that only the mesons $M_{a}^{\prime}$ become the dynamical degree of freedom in the SUSY breaking sectors together with singlets $S_{a}^{\prime}$ and $S$.

At $T \gtrsim \mu$, the scalar potential of $S$ and the messenger leptons, $\Phi_{l}$ and $\bar{\Phi}_{l}$, is approximately given by

$$
\begin{aligned}
V_{T} \simeq & V_{T=0}+\frac{1}{8}\left(3 g_{2}^{2}+g_{Y}^{2}+2 \kappa^{2}\right) T^{2}\left(\left|\Phi_{l}\right|^{2}+\left|\bar{\Phi}_{l}\right|^{2}\right) \\
& +\frac{5}{8} \lambda^{2} T^{2}|S|^{2}+\frac{5}{4} T^{2}|\kappa S-M|^{2}
\end{aligned}
$$

Here, $g_{2}$ and $g_{Y}$ are $S U(2)_{L}$ and $U(1)_{Y}$ gauge coupling constants, respectively. The thermal corrections by the SSM gauge interactions lift the scalar potential of the messengers. The messenger quarks, $\Phi_{d}$ and $\bar{\Phi}_{d}$, have larger thermal mass terms than the messenger leptons due to the $S U(3)_{C}$ interaction, though they are omitted in Eq. (17). The field values of the messengers are zero at the minimum of the scalar potential. The SUSY-invariant vacuum is hidden due to the thermal correction.

In addition, the interaction of mesons $M_{a}^{\prime}$ with $S$ makes the minimum around $S=0$ in the thermal potential. Since the messenger masses are given as $M-\kappa S$, the messengers would make a local minimum around $S \simeq M / \kappa$. However, the local minimum does not appear as far as $\lambda \gtrsim \kappa$, and the global minimum of the potential at $S \simeq 0$ and $\Phi_{d / l}=\bar{\Phi}_{d / l}=0$ is close to the SUSY-breaking vacuum at zero temperature 1 .

\footnotetext{
${ }^{1}$ Exactly speaking, when $M / \kappa \gg \lambda \mu$, the approximation in Eq. (17) is not valid and a local minimum around $S \simeq M / \kappa$ appears at $T \gtrsim \mu$. The thermal potential by mesons and singlets cannot lift the potential of $S$ much enough to hide the local minimum. If the subcritical bubbles of the local minimum are created
} 
When the temperature falls below $\sim \mu$, the stability of the SUSY-breaking vacuum becomes weaker. The SUSY true vacuum starts to appear, since the thermal potential by the SSM gauge interactions cannot hide it. It is found from Eq. (17) that the critical temperature $T_{c}$, at which the SUSY-breaking and SUSY-invariant vacua are degenerate, is $\lesssim \sqrt{8 \kappa / 3 g_{2}^{2}} \times \mu$. In addition, mesons $M_{a}^{\prime}$ and the singlets $S_{a}^{\prime}$ are gradually decoupled from the thermal bath so that the interaction of the messengers become relatively stronger. Notice here that the masses of the mesons and the singlets are $\sqrt{\lambda} \mu$.

After the mesons are decoupled, the first term in the second line of Eq. (17) disappears so that $S$ moves from $S \simeq 0$ toward $M / \kappa\left(S \simeq\left(4 \pi^{2}(T / \mu)^{2} \kappa^{2} / \lambda^{3}\right) \times(M / \kappa)\right)$. If $S$ approaches close to $M / \kappa, S$ and messengers would roll into the SUSY-invariant vacuum. Thus, $\kappa$ should be suppressed so that the universe is trapped in the SUSY-breaking vacuum.

Now we estimate the thermal transition rate from the SUSY-breaking to SUSYinvariant vacua. In the case of a finite temperature, the transition rate is determined by the three-dimensional Euclidean action $S_{3}$ as [16]

$$
\Gamma_{3} \simeq T^{4}\left(\frac{S_{3}}{2 \pi T}\right)^{3 / 2} e^{-\frac{S_{3}}{T}}
$$

Most of the present universe remains in the false (SUSY-breaking) vacuum when $S_{3} / T \gtrsim 230$ [16, 17].

When evaluating $S_{3}$, we choose a bounce solution whose path is from the SUSYbreaking vacuum $\left(S \sim 0\right.$ and $\left.\Phi_{d} \bar{\Phi}_{d}=\Phi_{l} \bar{\Phi}_{l}=0\right)$ to the SUSY-invariant vacuum $(S=M / \kappa$, $\Phi_{d} \bar{\Phi}_{d}=0$ and $\left.\Phi_{l} \bar{\Phi}_{l}=\mu^{2} / \kappa\right)$. The SUSY-invariant vacuum with $\Phi_{d} \bar{\Phi}_{d} \neq 0$ suffers from the stronger thermal correction due to the $S U(3)_{C}$ interaction as mentioned above, and it is more hidden at the low temperatures than the vacuum with $\Phi_{d} \bar{\Phi}_{d}=0$ and $\Phi_{l} \bar{\Phi}_{l}=\mu^{2} / \kappa$. This is a reason why we consider the above vacuum transition.

The $O(3)$ symmetric Euclidean action is given by

$$
S_{3}\left[\phi_{i}(r)\right]=4 \pi \int_{0}^{\infty} d r r^{2}\left[\sum_{i=1}^{2} \frac{k_{i}}{2}\left(\frac{d \phi_{i}}{d r}\right)^{2}+\left(V_{T}\left(\phi_{i}\right)-V_{T}\left(\phi_{i}^{f}\right)\right)\right] .
$$

by thermal hopping [15] and they survives at $T \lesssim \mu$, the phase transition to the true vacuum would be efficient. In this paper we are interested in smaller $M / \kappa \mu$, which leads to the light gravitino. Then, we do not discuss this case furthermore. 

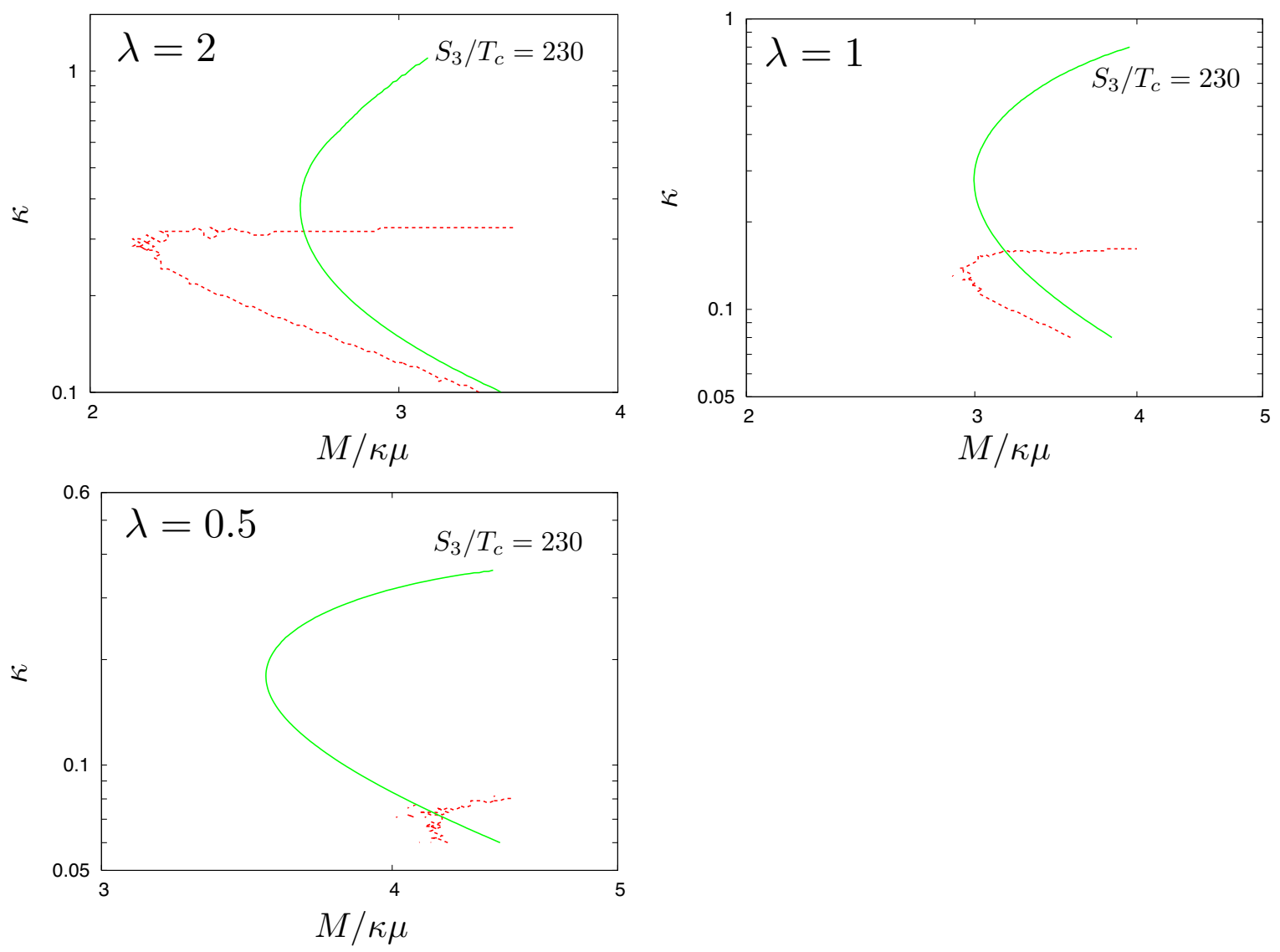

Figure 2: Solid (green) lines are for $S_{3} / T_{c}=230$ in plans of $\kappa$ and $M / \kappa \mu$ for $\lambda=2,1,0.5$. In regions except for the right-handed sides of dashed (red) lines, the SUSY-breaking vacuum becomes unstable in lower temperature than $\sim \mu$, at which the universe rolls down to the SUSY-invariant vacuum.

Here, $V_{T}\left(\phi_{i}^{f}\right)$ is the false vacuum energy at $T$. The scalar potential is almost flat around the critical temperature $T_{c}$. Then, we assume in evaluating $S_{3}$ that $V_{T}\left(\phi_{i}\right)$ is dominated by the zero-temperature potential, while we derive $T_{c}$ from the potential including thermal corrections. We use the formula for the thermal potential given in Ref. [18]. We estimate $S_{3}$ by the numerical method in Ref. [12], again.

In Fig. 2 we show that contour plots for $S_{3} / T_{c}=230$ on planes of $\kappa$ and $M / \kappa \mu$ for $\lambda=2,1,0.5$ by solid (green) lines. The left-handed sides of the lines, in which $S_{3} / T_{c}<230$, are disfavored. It is found from the triangle approximation that $S_{3}$ is approximately given by $8 \pi / 3(M / \kappa \mu)^{3} \mu$ in the regions away from the lines to the right-handed sides. 


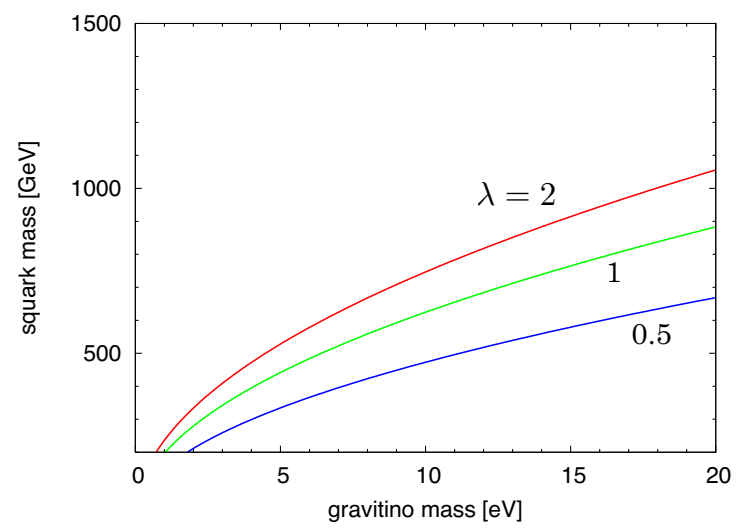

Figure 3: Squark mass upperbounds as functions of the gravitino mass for $\lambda=0.5,1,2$ from the stability of the SUSY-breaking vacuum in the thermal bath of the early universe.

In addition, in regions except for the right-handed sides of dashed (red) lines, the SUSY-breaking false vacuum becomes destabilized in lower temperature than $\sim \mu$ so that the universe rolls down to the SUSY-invariant vacuum. When $\kappa$ is larger, the messengers may destabilize the false vacuum as mentioned above. This excludes broad regions in the parameter space.

The stability of the SUSY-breaking vacuum in the thermal bath of the early universe gives a stronger constraint on $M / \kappa \mu$. We translate it to the squark mass upperbounds as functions of the gravitino mass for $\lambda=0.5,1,2$ in Fig. 3. When $m_{3 / 2}<16 \mathrm{eV}$, the squark masses have to be smaller than $1(0.8,0.6) \mathrm{TeV}$ for $\lambda=2(1,0.5)$. It is found after taking the mass functions $f$ and $g$ and the renormalization-group effects into consideration that the squark mass $m_{\tilde{q}}<1 \mathrm{TeV}$ corresponds to the gluino mass $m_{\tilde{g}} \lesssim 1 \mathrm{TeV}$ in our model.

Notice that we imposed $S_{3} / T>230$ at $T=T_{c}$ while using the zero-temperature scalar potential in the evaluation of $S_{3}$. If we evaluate $S_{3}$ as a function of $T$ using the potential including thermal correction and impose $S_{3} / T>230$ for $T \leq T_{c}$, the constraints on the squark masses would be weakened. We consider from studies of some sample points in our model that the correction on the squark mass upperbounds is at most $O(10) \%$. 


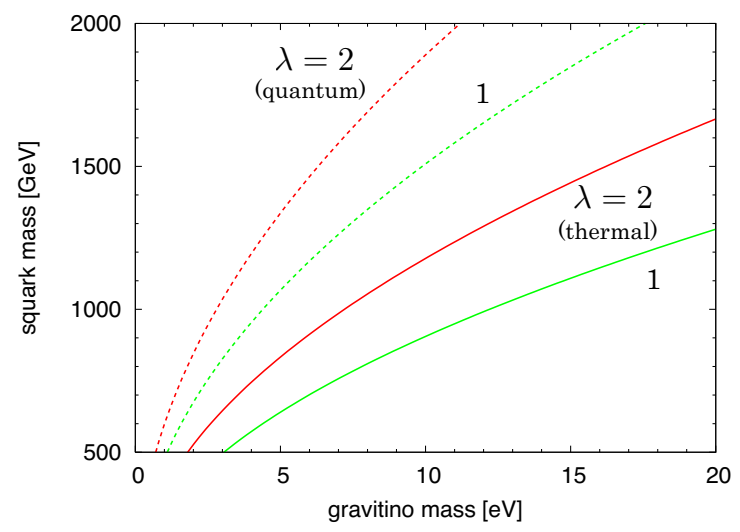

Figure 4: Squark mass upperbounds as functions of gravitino mass for $\lambda=1$, 2. Here, we assume the messenger model with $\mathbf{1 0}+\mathbf{1 0}^{\star}$. Dashed (solid) lines come from stability of the SUSY-breaking vacuum against quantum (thermal) transition.

\section{Conclusions and discussion}

In this paper we have discussed the vacuum transitions at zero temperature and at high temperatures in the early universe by assuming the IYIT model for the gauge mediation. This model accommodates a gauge mediation with the light gravitino of mass $m_{3 / 2} \lesssim 16 \mathrm{eV}$, but the SUSY particle masses in the SSM model are bounded from above to maintain the thermal and quantum stabilities of the SUSY-breaking false vacuum. We have, in fact, found that $m_{\tilde{q}} \lesssim 1 \mathrm{TeV}$ and $1.5 \mathrm{TeV}$ for the thermal and quantum stabilities, respectively, when imposing $m_{3 / 2} \lesssim 16 \mathrm{eV}$.

In the rest, we discuss possibilities to weaken the bounds. The bounds we have derived in this paper are based on the minimal messenger model, in which $\mathbf{5}+\mathbf{5}^{\star}$ in the $S U(5)_{\text {GUT }}$ are introduced. An alternative choice of the messengers is a pair of messengers belonging to $10+10^{\star}$. In Fig. 4, the upperbounds for the squark masses are shown as functions of the gravitino mass in the messenger model with $\mathbf{1 0}+\mathbf{1 0}^{\star}$. The solid (dashed) lines come from the thermal (quantum) stability for $\lambda=1,2$. While increase of the number of the messengers makes stability of the SUSY-breaking vacuum weaker, the gaugino and sfermion masses are enhanced by 3 and $\sqrt{3}$, respectively. It is found that the the upperbound on squark masses from the thermal stability is increased to $1.5 \mathrm{TeV}$. This corresponds to the upperbound on the gluino mass $m_{\tilde{g}} \lesssim 2.0 \mathrm{TeV}$. 
For the SUSY-breaking sector, we can extend the hidden gauge symmetry to $S p(2 N)$ gauge group. The number of mesons and singlets, $M_{a}^{\prime}$ and $S_{a}^{\prime}$, is $2 N^{2}+3 N$, while the

Yukawa coupling of mesons with $S$ is suppressed by $1 / \sqrt{N}$. Then, $m_{S}^{2}$ is enhanced by $N^{1 / 2}$, and the larger $N$ stabilizes the SUSY-breaking vacuum more strongly. In addition, the mass of mesons and singlets is scaled by $N^{-1 / 4}$. When $N \gg 1$, the thermal correction to the scalar potential by the light mesons is more efficient at low temperatures than in the $S U(2)$ case. Thus, large $N$ model allows larger squark masses than the original $S U(2)$ model.

Finally, we should stress that an improvement of the gravitino mass bound [1] by a factor of 2 may give a serious problem for the present gauge mediation model.

\section{Acknowledgement}

This work was supported by World Premier International Center Initiative (WPI Program), MEXT, Japan. The works of JH and TTY was also supported in part by the Grantin-Aid for Science Research, Japan Society for the Promotion of Science (No. 20244037 and No. 2054252 for JH and No. 1940270 for TTY).

\section{References}

[1] M. Viel, J. Lesgourgues, M. G. Haehnelt, S. Matarrese and A. Riotto, Phys. Rev. D 71 (2005) 063534.

[2] M. Kawasaki, K. Kohri and T. Moroi, Phys. Lett. B 625 (2005) 7 arXiv:astro-ph/0402490; Phys. Rev. D 71 (2005) 083502 arXiv:astro-ph/0408426].

[3] M. Kawasaki, F. Takahashi and T. T. Yanagida, Phys. Lett. B 638 (2006) 8 arXiv:hep-ph/0603265]; Phys. Rev. D 74 (2006) 043519 arXiv:hep-ph/0605297; M. Endo, F. Takahashi and T. T. Yanagida, Phys. Lett. B 658 (2008) 236 arXiv:hep-ph/0701042].

[4] M. Dine, W. Fischler and M. Srednicki, Nucl. Phys. B 189 (1981) 575; S. Dimopoulos and S. Raby, Nucl. Phys. B 192 (1981) 353; See also references in G. F. Giudice and R. Rattazzi, Phys. Rept. 322 (1999) 419 [arXiv:hep-ph/9801271]. 
[5] J. Hisano and Y. Shimizu, Phys. Lett. B 655 (2007) 269 [arXiv:0706.3145 [hep-ph]].

[6] J. Hisano, M. Nagai, M. Senami and S. Sugiyama, Phys. Lett. B 659 (2008) 361 arXiv:0708.3340 [hep-ph]].

[7] K. I. Izawa and T. Yanagida, Prog. Theor. Phys. 95 (1996) 829 arXiv:hep-th/9602180]; K. A. Intriligator and S. D. Thomas, Nucl. Phys. B 473 (1996) 121 arXiv:hep-th/9603158].

[8] Z. Chacko, M. A. Luty and E. Ponton, JHEP 9812 (1998) 016 arXiv:hep-th/9810253.

[9] S. P. Martin, Phys. Rev. D 55 (1997) 3177 [arXiv:hep-ph/9608224].

[10] H. Murayama and Y. Nomura, Phys. Rev. D 75 (2007) 095011 arXiv:hep-ph/0701231.

[11] S. R. Coleman, Phys. Rev. D 15 (1977) 2929 [Erratum-ibid. D 16 (1977) 1248]; C. G. Callan and S. R. Coleman, Phys. Rev. D 16 (1977) 1762.

[12] T. Konstandin and S. J. Huber, JCAP 0606 (2006) 021 [arXiv:hep-ph/0603081].

[13] A. Kusenko, Phys. Lett. B 358 (1995) 47 [arXiv:hep-ph/9506386].

[14] M. J. Duncan and L. G. Jensen, Phys. Lett. B 291 (1992) 109.

[15] M. Gleiser, E. W. Kolb and R. Watkins, Nucl. Phys. B 364 (1991) 411.

[16] A. D. Linde, Nucl. Phys. B 216 (1983) 421 [Erratum-ibid. B 223 (1983) 544].

[17] A. H. Guth and E. J. Weinberg, Phys. Rev. D 23 (1981) 876.

[18] L. Dolan and R. Jackiw, Phys. Rev. D 9 (1974) 3320. 